\title{
Surgical and sensory outcomes in patients with intermittent exotropia according to preoperative refractive error
}

\author{
Ye Jin Ahn ${ }^{1}$ - Yoo Yeon Park ${ }^{1} \cdot$ Yeon Woong Chung ${ }^{2}$ Shin Hae Park ${ }^{1}$ Sun Young Shin ${ }^{1}$
}

Received: 1 May 2018 / Accepted: 8 February 2019 / Published online: 1 April 2019

(c) The Royal College of Ophthalmologists 2019

\begin{abstract}
Background/Objectives To analyze the surgical and sensory outcomes of intermittent exotropia according to refractive errors and the factors associated with surgical success.

Subjects/Methods A total of 326 children were divided into three groups according to preoperative refractive error; hyperopic eyes with $\mathrm{SE} \geq+2 \mathrm{D}$ (hyperopic group), eyes with $\mathrm{SE}$ between $-1 \mathrm{D}$ and $+2 \mathrm{D}$ (emmetropic group), and myopic eyes with $\mathrm{SE} \leq-1 \mathrm{D}$ (myopic group). The surgical outcomes and the sensory outcomes measured by near and distant stereoacuity were compared among the three groups.

Results The surgical success rate in hyperopic group was significantly lower compared to myopic group at last follow-up $(P=0.012)$. Preoperative near stereopsis was not different among three groups, however, the distance stereopsis was significantly deteriorated in the hyperopic group compared to the other two groups (Titmus; $P=0.168$, FD2; $P<0.001$, DR; $P=0.048$ ). There was postoperative improvement in both near and distant stereopsis in all three groups (Titmus; $P=0.009$, FD2; $P=0.021$, DR; $P=0.036$ ) and no significant difference was found in the postoperative distant stereopsis among the three groups.

Conclusions Preoperative refractive error is a prognostic factor of surgical success in patients with intermittent exotropia. Patients with hyperopia achieved less favorable surgical outcome compared to myopic patients. The preoperative distant stereoacuity was decreased in hyperopic patients compared to myopic patients, which eventually improved after surgery and showed no significant difference at postoperative measurements.
\end{abstract}

\section{Introduction}

Intermittent exotropia is one of the most common strabismus entities worldwide accounting for $25 \%$ of all strabismus and $1 \%$ of the general population [1]. Surgical treatment has been conventionally offered when the estimated period of misalignment is greater than half of the time, or when stereoacuity is declining, or misaligned appearance is causing social problems. However, the indications, timing, and choice of procedure remains

Sun Young Shin

eyeshin@ catholic.ac.kr

1 Department of Ophthalmology and Visual Science, Seoul St. Mary's Hospital, College of Medicine, The Catholic University of Korea, Seoul, Republic of Korea

2 Department of Ophthalmology, S St. Vincent's Hospital, College of Medicine, The Catholic University of Korea, Seoul, Republic of Korea controversial and the success rate is also highly variable ranging from 42 ot $81 \%$ [2-5]. There have been efforts to evaluate the association between different types of refractive errors in relation to the development of intermittent exotropia [6, 7]. Children with myopia had greater risk in developing exotropia than those without significant ametropia, which explains the high prevalence of exotropia in Asia, where the prevalence of myopia is much higher [8]. Nevertheless, we encounter hyperopic or ametropic children with concomitant exotropia who requires surgical intervention in our daily practice, and there is a lack of consensus on the effect of different types of refractive errors on the surgical success.

The purpose of this study was to analyze the surgical outcomes of intermittent exotropia according to refractive errors and the factors associated with surgical success. In addition, we evaluated the changes in stereoacuity at both near and far and their effect on the surgical outcome. 


\section{Methods}

This observational case series was approved by the institutional review board of Seoul Saint Mary's hospital and the study protocol followed the guidelines of the Declaration of Helsinki. We retrospectively reviewed the medical records of children who received surgery for intermittent exotropia by a single surgeon (SYS) at Seoul St. Mary's Hospital from 2009 to 2016.

All children underwent overall ophthalmic examination at initial visit, including slit-lamp biomicroscopy and fundus photography to exclude any structural abnormalities. The following preoperative characteristics were recorded from the patients' charts: age at onset, age at surgery, the type of surgery, best-corrected visual acuity (BCVA), refractive error, preoperative motor alignment at distance and near, stereoacuity at distance and near. Refractive error was measured after topical instillation of $1 \%$ cyclopentolate and $0.5 \%$ mydriacyl in younger children and by manifest refraction in relatively cooperative older children. All refractive error was reported in terms of spherical equivalent (SE), calculated as the sphere plus half a cylinder. Anisometropia was defined as a spherical or cylindrical difference of at least 2 diopters (D) between the two eyes, and amblyopia was defined as a difference of two or more lines between monocular visual acuities. Ocular alignment was tested by prism alternative cover testing at $4 \mathrm{~m}$ fixation and $30 \mathrm{~cm}$ fixation in older children and for preverbal children, the Krimsky or Hirschberg light reflex test was done. If the distant angle of exodeviation was greater than 10 prism diopters (PD) compared to the near angle, the angle was remeasured after 1-h monocular occlusion of the nondominant eye. Also the angle of deviation was measured in the nine cardinal positions of gaze for determination of the degree of incomitance. Sensory test was performed using the Titmus test (Stereo Optical Inc.,Chicago, IL, USA) for near and Frisby-Davis test (FD2) and Distant Randot test (DR) for far. If the patient failed the largest disparity, stereoacuity was recorded as nil and was assigned a value of $6000^{\prime \prime}$ for Titmus test, $400^{\prime \prime}$ for FD2, and $800^{\prime \prime}$ for DR.

Postoperative status of motor alignment at distance and near and stereoacuity at distance and near were examined at 3,6 , and 12 months after the surgery. Patients who followed up over 12 months, the data from the last visit was also collected. Patients with forms of strabismus other than intermittent exotropia (e.g., constant exotropia, paralytic strabismus, vertical strabismus, oblique muscle dysfunction, A-V pattern strabismus) or a follow-up visit $<12$ months were not included in the study. Patients with severe amblyopia, developmental delay, any type of neurologic impairment or other diseases of the visual pathways, and previous extraocular muscle surgery were also excluded.

All patients were classified into three groups, according to preoperative cycloplegic refraction, which maintained throughout follow-up period; hyperopic eyes with $\mathrm{SE} \geq+2 \mathrm{D}$ in both eyes (hyperopic group), eyes with $\mathrm{SE}$ between -1D and $+2 \mathrm{D}$ in both eyes (emmetropic group), and myopic eyes with $\mathrm{SE} \leq-1 \mathrm{D}$ in both eyes (myopic group). Surgery was considered when there was deterioration in the frequency of the manifest phase of the intermittent exotropia or the angle of deviation measured at least 15PD. The surgical outcome was considered successful when the distant deviation with correction was $\leq 5 \mathrm{PD}$ esodeviation and $\leq 10 P D$ exodeviation. Reoperation was considered a failure and patients who received reoperation were included until the last follow-up visit before reoperation.

Patients with hyperopia of $>+3 \mathrm{D}$, myopia of $\geq-1.5 \mathrm{D}$, or astigmatism of $\geq+1.50 \mathrm{D}$ or more were prescribed glasses before a final surgical decision was made. In patients with hyperopia, glasses of $\sim+1$ to $+1.5 \mathrm{D}$ less than the full cycloplegic hyperopic refraction were given for optimal correction of visual acuity.

For statistical analysis, one-way analysis of variance (ANOVA), Chi-square test, and repeated measures ANOVA were used for the comparison of three groups. In addition, multivariate logistic regression was performed to determine associations with surgical success and preoperative clinical characteristics. However, because of the large number of variables, only those with a univariate association near significance $(P<0.2)$ were included. Direct comparison between means was assessed using a Student' $t$-test. $\mathrm{P}$ value of $<0.05$ was accepted as significant. All statistical data were analyzed using SPSS Statistics 19.0 software (IBM Corporation, Armonk, NY, USA).

\section{Results}

A total of 326 children were included in the study. The hyperopic group consisted of 34 subjects, 170 in the emmetropic group and 122 in the myopic group. The age at onset was significantly different among groups. Patients in myopic group were significantly older when their exodeviation was first reported compared to patients in the other two groups $(P<0.001)$. However, neither the age at operation nor the time from diagnosis to surgery differed between groups. The patients in hyperopic group had more anisometropia compared to the other two groups $(P<$ $0.001)$. There were statistically significant differences in the preoperative distant stereopsis among groups; patients in hyperopic group showed worse distant stereoacuity both in the FD2 and DR tests compared to the emmetropic and myopic patients $(P=0.001$ and $P=0.048$, respectively). Gender, preoperative angle of deviation, preoperative near stereopsis, type of exotropia, type of surgery and the followup period after surgery did not statistically differ between the three groups. Data are summarized in Table 1. 
Table 1 Demographic characteristics of patients according to preoperative refractive errors

\begin{tabular}{|c|c|c|c|c|}
\hline & $\begin{array}{l}\text { Hyperopic group } \\
(n=34)\end{array}$ & $\begin{array}{l}\text { Emmetropic group } \\
(n=170)\end{array}$ & $\begin{array}{l}\text { Myopic group } \\
(n=122)\end{array}$ & $P$-value \\
\hline Gender (male, $N$ ) & 11 & 80 & 62 & $0.207^{\dagger}$ \\
\hline Age at onset (years) & $3.56 \pm 7.81$ & $4.53 \pm 1.72$ & $6.48 \pm 2.42$ & $<0.001 *$ \\
\hline Age at operation (years) & $6.05 \pm 2.25$ & $5.72 \pm 1.87$ & $7.73 \pm 2.58$ & $0.07 *$ \\
\hline $\begin{array}{l}\text { Time from diagnosis to } \\
\text { surgery (months) }\end{array}$ & $27.64 \pm 23.22$ & $20.83 \pm 17.70$ & $22.48 \pm 19.99$ & $0.149 *$ \\
\hline Presence of anisometropia & 11 & 2 & 30 & $<0.001^{\dagger}$ \\
\hline $\begin{array}{l}\text { Preoperative refractive error } \\
\text { (D) }\end{array}$ & $\begin{array}{l}2.93 \pm 1.08 \text { (range: } \\
2 \sim 5)\end{array}$ & $\begin{array}{l}0.34 \pm 0.59 \text { (range: } \\
-0.875 \sim 1.75 \text { ) }\end{array}$ & $\begin{array}{l}-2.82 \pm 1.90 \\
\text { (range: }-1 \sim \\
-4.375 \text { ) }\end{array}$ & $<0.001 *$ \\
\hline $\begin{array}{l}\text { Preoperative distant deviation } \\
\text { (PD) }\end{array}$ & $24.26 \pm 8.07$ & $22.68 \pm 4.59$ & $22.64 \pm 4.95$ & $0.315^{*}$ \\
\hline $\begin{array}{l}\text { Preoperative near deviation } \\
\text { (PD) }\end{array}$ & $25.56 \pm 8.95$ & $23.09 \pm 6.72$ & $24.84 \pm 6.46$ & $0.166^{*}$ \\
\hline \multicolumn{5}{|c|}{ Preoperative distant stereopsis (arsec) } \\
\hline FD2 & $215.00 \pm 189.88$ & $77.03 \pm 129.92$ & $65.17 \pm 116.61$ & $0.001 *$ \\
\hline DR & $357.04 \pm 348.66$ & $206.79 \pm 276.49$ & $261.79 \pm 341.63$ & $0.048 *$ \\
\hline $\begin{array}{l}\text { Preoperative near stereopsis } \\
(\operatorname{arsec})\end{array}$ & $513.85 \pm 780.52$ & $287.64 \pm 673.08$ & $222.14 \pm 555.63$ & $0.168^{*}$ \\
\hline Type of exotropia & & & & $0.393^{\dagger}$ \\
\hline Basic & 31 & 156 & 110 & \\
\hline Divergence excess & 0 & 4 & 0 & \\
\hline Pseudodivergence & 2 & 10 & 6 & \\
\hline Convergence insufficiency & 1 & 0 & 6 & \\
\hline Type of surgery & & & & $0.979^{\dagger}$ \\
\hline BLR recession & 21 & 144 & 102 & \\
\hline ULR recession & 12 & 24 & 18 & \\
\hline $\mathrm{R}$ and $\mathrm{R}$ & 1 & 2 & 2 & \\
\hline $\begin{array}{l}\text { Follow-up period after } \\
\text { surgery (months) }\end{array}$ & $40.35 \pm 27.90$ & $39.62 \pm 25.82$ & $35.54 \pm 22.19$ & $0.550 *$ \\
\hline
\end{tabular}

Hyperopic group ( $\geq+2$ diopters); Emmetropic group ( $-1<$ diopters $<+2)$; Myopic group ( $\leq-2$ diopters)

$N$ number, $D$ diopters, $P D$ prism diopters, $F D 2$ Frisby-Davis test, $D R$ Distant Randot test, $B L R$ bilateral lateral rectus muscle, $U L R$ unilateral lateral rectus muscle, $R$ and $R$ lateral rectus muscle recession and medial rectus muscle resection

* $P$-value by one-way ANOVA

${ }^{\dagger} P$-value by Chi-square test

Table 2 describes preoperative factors for risk of surgical failure at the last follow-up. Univariate analysis revealed preoperative refractive error status $(P=0.123)$ and preoperative near stereopsis $(P=0.166)$ were trended toward an association with a surgical failure. However, multivariate analysis revealed that only preoperative refractive error was a truly significant association $(P=0.011)$. Neither the presence of anisometropia, nor the preoperative distant stereopsis showed significant association.

Figure 1 shows surgical success rates at each postoperative follow-up period. The success rate did not differ at postoperative 3, 6, and 12 months between three groups. However, at the last follow-up, statistically significant difference was found between the three groups $(P=0.013)$. The myopic group $(77.8 \%)$ had higher surgical success rate than emmetropic group (59.4\%) and hyperopic group (64.0\%), but only the difference between myopic group and the hyperopic group was statistically significant $(P=0.012)$.

There was significant difference in preoperative and postoperative stereopsis improvement between the three groups (Titmus; $P=0.009$, FD2; $P=0.021$, DR; $P=0.036$ by repeated-measures ANOVA). Patients in hyperopic group had worse preoperative stereopsis compared to the other two groups, however, only the distant stereopsis showed statistical significance (Titmus; $P=0.168$, FD2; $P<0.001$, DR; $P=0.048)$. Postoperatively, the near and distant stereopsis was still worse in hyperopic group than the other two groups, however, the difference was not significant at postoperative 6,12 months and at last follow-up, among the three groups (Table 3). 
Table 2 Comparison of preoperative factors for risk of surgical failure at last follow-up

\begin{tabular}{|c|c|c|c|}
\hline & Success $(n=249)$ & Failure $(n=77)$ & $\begin{array}{l}P \text {-value } \\
\text { (multivariate analysis) }\end{array}$ \\
\hline Gender (male, $N$ ) & 115 & 32 & 0.344 \\
\hline Age at onset (years) & $4.78 \pm 4.40$ & $4.00 \pm 2.13$ & 0.423 \\
\hline Age at operation (years) & $6.20 \pm 2.61$ & $5.26 \pm 1.86$ & 0.565 \\
\hline Time from diagnosis to surgery (months) & $23.84 \pm 22.56$ & $20.73 \pm 12.32$ & 0.463 \\
\hline Preoperative refractive error (SE, D) & $-1.27 \pm 1.88$ & $-0.23 \pm 2.58$ & $0.123(0.011)$ \\
\hline Presence of anisometropia & 46 & 17 & 0.998 \\
\hline Preoperative distant deviation (PD) & $22.68 \pm 5.18$ & $23.68 \pm 6.58$ & 0.738 \\
\hline Preoperative near deviation (PD) & $23.72 \pm 7.23$ & $25.83 \pm 6.75$ & 0.432 \\
\hline \multicolumn{4}{|l|}{ Preoperative distant stereopsis (arsec) } \\
\hline FD2 & $111.29 \pm 158.65$ & $78.13 \pm 126.31$ & 0.907 \\
\hline $\mathrm{DR}$ & $253.89 \pm 312.22$ & $374.78 \pm 331.29$ & 0.250 \\
\hline Preoperative near stereopsis (arsec) & $271.83 \pm 599.72$ & $441.67 \pm 877.54$ & $0.166(0.052)$ \\
\hline \multicolumn{4}{|l|}{ Type of exotropia } \\
\hline Basic & 235 & 76 & 0.878 \\
\hline Divergence excess & 1 & 1 & 0.611 \\
\hline Pseudodivergence & 9 & 0 & 0.668 \\
\hline Convergence insufficiency & 4 & 0 & 0.871 \\
\hline \multicolumn{4}{|l|}{ Type of surgery } \\
\hline BLR recession & 169 & 63 & 0.962 \\
\hline ULR recession & 70 & 14 & 0.998 \\
\hline $\mathrm{R}$ and $\mathrm{R}$ & 10 & 0 & 0.946 \\
\hline Follow-up period after surgery (months) & $37.61 \pm 25.89$ & $41.75 \pm 22.15$ & 0.291 \\
\hline
\end{tabular}

$N$ number, $S E$ spherical equivalent, $D$ diopters, $P D$ prism diopters, $F D 2$ Frisby-Davis test, $D R$ Distant Randot test, $B L R$ bilateral lateral rectus muscle, $U L R$ unilateral lateral rectus muscle, $R$ and $R$ lateral rectus muscle recession and medial rectus muscle resection

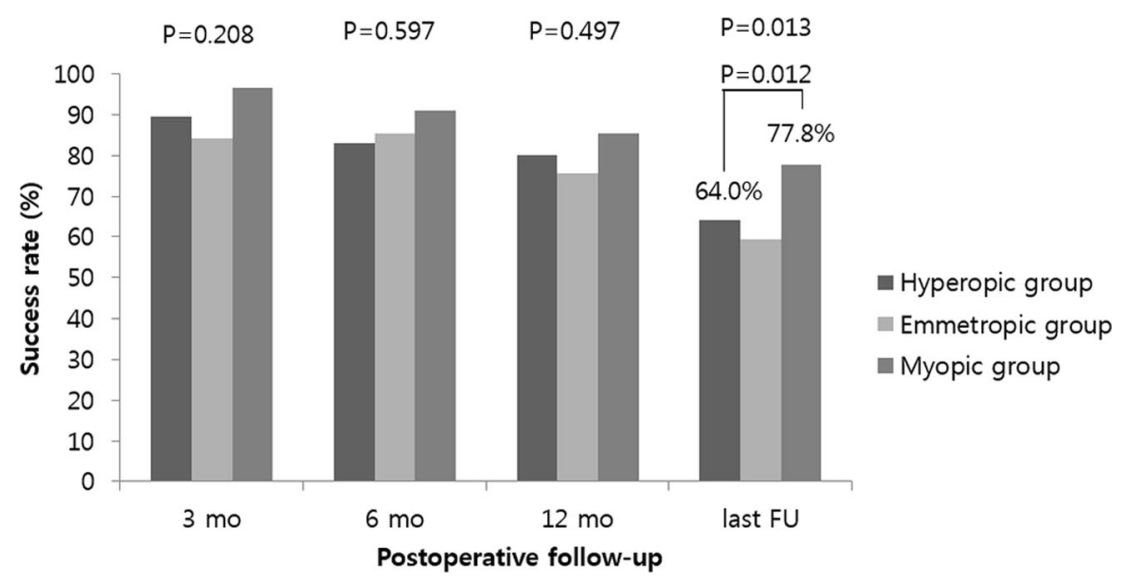

Fig. 1 The surgical success rates at postoperative 3, 6, 12 months and the last follow-up. There were no significant differences between three groups except for the last follow-up $(P=0.013)$. At the last follow-up, there were no significant differences between emmetropic group and

\section{Discussion}

In this study, the prognostic factor of surgical success in patients with intermittent exotropia was the preoperative refractive error. Patients with hyperopia achieved less the other two groups (hyperopic group; $P=0.111$, myopic group; $P=$ 0.091, respectively). However, hyperopic group (64.0\%) and myopic group $(77.8 \%)$ demonstrated statistically significant difference in the success rate $(P=0.012)$

favorable surgical outcomes compared to myopic patients. The preoperative distant stereoacuity was decreased in hyperopic patients compared to myopic patients, which eventually improved after surgery and showed no significant difference at postoperative measurements. 
Table 3 Comparison of pre- and postoperative stereopsis according to preoperative refractive errors

\begin{tabular}{lcccc}
\hline & Hyperopic group $(n=34)$ & Emmetropic group $(n=170)$ & Myopic group $(n=122)$ & $P$-value \\
\hline Preoperative stereopsis (arsec) & & & \\
Titmus test & $513.85 \pm 780.52$ & $287.64 \pm 673.08$ & $222.14 \pm 555.63$ & 0.168 \\
FD2 & $215.00 \pm 189.88$ & $77.03 \pm 129.92$ & $65.17 \pm 116.61$ & 0.001 \\
DR & $562.60 \pm 329.23$ & $206.79 \pm 276.49$ & $261.79 \pm 341.63$ & 0.048 \\
Postoperative 3 mo stereopsis (arsec) & & & \\
Titmus test & $338.57 \pm 770.79$ & $145.00 \pm 398.33$ & $101.67 \pm 133.31$ & 0.136 \\
FD2 & $120.00 \pm 172.85$ & $30.90 \pm 61.56$ & $43.29 \pm 89.96$ & 0.410 \\
DR & $357.04 \pm 348.66$ & $172.35 \pm 255.42$ & $203.89 \pm 277.74$ & 0.001 \\
Postoperative 6 mo stereopsis (arsec) & & & \\
Titmus test & $162.11 \pm 132.77$ & $138.02 \pm 113.06$ & $73.33 \pm 68.91$ & 0.090 \\
FD2 & $85.00 \pm 139.43$ & $23.50 \pm 11.05$ & $38.86 \pm 81.91$ & 0.438 \\
DR & $246.15 \pm 264.62$ & $158.24 \pm 225.16$ & $187.24 \pm 259.44$ & 0.496 \\
Postoperative 1 year stereopsis (arsec) & & & \\
Titmus test & $150.00 \pm 93.27$ & $131.31 \pm 380.24$ & $60.00 \pm 33.73$ & 0.310 \\
FD2 & $60.00 \pm 11.97$ & $36.11 \pm 73.57$ & $42.62 \pm 84.91$ & 0.425 \\
DR & $220.00 \pm 275.29$ & $141.80 \pm 223.49$ & $111.51 \pm 180.02$ & 0.336 \\
Stereopsis at final follow-up (arsec) & & & \\
Titmus test & $116.15 \pm 161.53$ & $73.27 \pm 65.87$ & $58.97 \pm 24.79$ & 0.056 \\
FD2 & $38.00 \pm 3.82$ & $16.82 \pm 7.51$ & $22.00 \pm 10.37$ & 0.353 \\
DR & $184.44 \pm 256.78$ & $82.56 \pm 43.33$ & $133.60 \pm 178.58$ & 0.100 \\
\hline
\end{tabular}

Hyperopic group ( $\geq+2$ diopters), Emmetropic group $(-1<$ diopters $<+2)$, Myopic group $(\leq-2$ diopters)

$F D 2$ Frisby-Davis test, $D R$ Distant Randot test, mo month

Titmus test; $P=0.009$, FD2; $P=0.021$, DR; $P=0.036$ by repeated-measures analysis of variance
Outcomes of surgical correction for intermittent exotropia have been studied since the 1960s [9]. In spite of the huge number of literatures, there is still lack of consensus on the success rates, criteria for successful outcome, as well as the predictive factors of surgical success. It can be presumed that increased age at surgery might be associated with good prognosis, because in older patients, more accurate measurements of the angle of deviation is possible, allowing more accurate decision on the amount of surgery. However, a number of previous studies have shown that age at surgery was not a significant prognostic factor [10-12]. This is consistent with our results, that neither the age at onset nor the age at surgery were associated with surgical success, even though our study included relatively younger patients than those studies. Considering the progressive nature of exotropia and delayed fusion would lead to irreversible sensory deficit, careful decision on the timing of surgery of exotropia must be made.

In our study, preoperative angle of deviation was not a predictive factor for successful surgery. This is similar to Richard and Parks [4], and Stoller et al. [13], who concluded that the preoperative angle had insignificant effect on the surgical outcome. On the other hand, Gezer et al. [10] reported that the preoperative deviation is a significant factor influencing both a favorable outcome $\left(r^{2}=0.12, P<\right.$ $0.001)$ and the efficacy of the surgery performed $\left(r^{2}=0.31\right.$, $P<0.001)$. Although preoperative angle of deviation was not associated significantly with surgical success in our study, special care should be given to preoperative angles in patients with exotropia. Other preoperative factors, such as gender, presence of anisometropia, type of exodeviation, type of surgery, preoperative stereoacuity at near and far, time from diagnosis to surgery, and follow-up period after surgery did not seem to increase the risk of surgical failure.

The only preoperative prognostic factor in surgery of intermittent exotropia was the preoperative refractive error in this study. Hyperopic children are at an increased risk for development of unilateral or bilateral amblyopia, esotropia and anisometropia [14, 15], Anisometropia was more frequently present in the hyperopic group in our study, however, anisometropia itself turned out to have no effect on the surgical success, which is supported by other studies $[11,16]$. There have been previous reports dealing with preoperative refractive errors and their influence on the surgical outcomes of intermittent exotropia. Lim et al. [17] found that refractive error was not associated with surgical prognosis. Grezer et al. [10] demonstrated that surgery performed on more myopic patients were less effective compared to patients with emmetropia or hyperopia. Nevertheless, the study used a greater response to surgery as an effective surgery, not defining surgical success, and those two variables are not interchangeable. Kim et al. [18] reported hyperopia was not an indicator for poor prognosis, instead, taking into account to the age effect, follow-up period after surgery and stereopsis improvement, hyperopia is rather a good prognostic factor. On the other hand, in the work of Zou et al. [19]. larger myopic refractive error 
correlated with surgical success, and more hyperopia correlated with lower success rates.

In this study, the success rate in the hyperopic group was significantly lower than the myopic group after the surgery for intermittent exotropia. The age at surgery and the follow-up period after surgery did not differ between groups, therefore, it can be considered that there was no age effect on the success rate. There have been explanations, that the prismatic effect of lenses prescribed to patients may alter the measured deviation of strabismus, resulting in increased tendency of under or overcorrection [20]. However, this effect begins to be clinically significant with lens powers $\geq+/-5 \mathrm{D}$, which was not taken into account in our study. Unlike the previous study, which included lower amount hyperopia, with SE higher than +1D [18], patients with SE exceeding $+2 \mathrm{D}$ in both eyes were classified as the hyperopic group in our study. In addition, as children undergo consistent emmetropization or myopic shift, the change in the refractive error status might have influenced the surgical outcome during the follow-up period. On the other hand, we included more hyperopic patients with persistent hyperopia during follow up period, which would explain why our result is different from the previous study.

Spectacle correction in children with hyperopia and exotropia can decrease the demand on the accommodative convergence and thus increase the exodeviation [21]. However, paradoxical resolutions of exotropia after spectacle correction of hyperopia was reported, which was explained by an improved fusional convergence with spectacles and a relatively low accommodative convergence over accommoation (AC/A) ratio [22]. Chung et al. [23] reported that, exotropic patients with hyperopia showed an increase in angle of deviation after spectacle correction. The worsening of exotropia was more remarkable in the partially corrected group (pure hyperopia) than in the fully corrected group (hyperopic astigmatism or amblyopia). The authors presumed the measurement of increased exotropia due to hyperopia correction might improve the surgical outcome. The patients in our study had mainly hyperopia than hyperopic astigmatism and the majority was prescribed with undercorrected spectacles for hyperopic refractive error. The worse surgical outcome of hyperopic patients with partial correction might indicate, hyperopic refractive error is a poor prognostic factor for intermittent exotropia surgery.

Most patients with intermittent exotropia are known to have good near stereoacuity, which remains stable preoperatively and postoperatively $[21,24]$. Holmes et al. [25] also believed that near stereoacuity is stable and surgery cannot be considered as an effective way to restore near stereoacuity in intermittent exotropia. Whereas, distant stereoacuity is known to correlate better with the course of intermittent exotropia than near stereoacuity and has been suggested to be applied in monitoring of deterioration or determining the proper time for surgery [26-28]. Previous studies have demonstrated that, there was a significant improvement in distance stereoacuity after surgery, although it was damaged seriously before the operation $[24,29]$. In the present study, the near stereoacuity with the Titmus test improved after surgery in all groups, but the difference between groups was not statistically significant. It is generally found that the maturation of stereopsis is nearly completed at an age $3-5$ years $[30,31]$. We believe the decreased near stereoacuity before surgery and the improvement after surgery may be due to the relatively low age of our subjects. On the other hand, the distant stereoacuity measured with FD2 and DR was significantly deteriorated, especially in the hyperopic group, which however, improved after the surgery.

The limitations of our study are as follows: first, intergroup variability exists, that the sample size of the hyperopic group was much smaller than the other two groups. Second, the follow-up period was relatively short. Third, direct comparisons with other studies are limited by differences in the baseline demographics, statistical analysis, and definition of success. This might limit the predictive value of our study, when applied to other patient cohort. Further study dealing with a longer follow-up period is required to confirm the results which were suggested in our study.

In conclusion, the results in this study indicate that preoperative refractive error is a predictor for favorable outcome of surgical treatment in cases of intermittent exotropia. Surgery performed on patients with hyperopia is expected to have lower success rates than surgery performed on patients with myopia although the preoperative distant stereoacuity was improved after the surgery.

\section{Summary}

\section{What was known before}

- There is lack of consensus on the prognostic factors for intermittent exotropia surgery

- Preoperative refractive error is one of them, but previous studies leads to various results

\section{What this study adds}

- Hyperopic patients with intermittent exotropia achieved less favorable surgical outcome compared to myopic patients

- However, the sensory outcome was not affected by the preoperative refractive error. 
Acknowledgements We thank all the patients for participating in this study. All authors have completed and submitted the ICMJE form for disclosure of potential conflicts of interest.

\section{Compliance with ethical standards}

Conflict of interest The authors declare that they have no conflict of interest.

Publisher's note: Springer Nature remains neutral with regard to jurisdictional claims in published maps and institutional affiliations.

\section{References}

1. Rutstein RP, Corliss DA. The clinical course of intermittent exotropia. Optom Vis Sci. 2003;80:644-9.

2. Ekdawi NS, Nusz KJ, Diehl NN, Mohney BG. Postoperative outcomes in children with intermittent exotropia from a population-based cohort. J AAPOS. 2009;13:4-7.

3. Hardesty HH, Boynton JR, Keenan JP. Treatment of intermittent exotropia. Arch Ophthalmol. 1978;96:268-74.

4. Richard JM, Parks MM. Intermittent exotropia. Surgical results in different age groups. Ophthalmology. 1983;90:1172-7.

5. Clarke WN, Noel LP. Surgical results in intermittent exotropia. Can J Ophthalmol. 1981;16:66-9.

6. Tang SM, Chan RY, Bin Lin S, Rong SS, Lau HH, Lau WW, et al. Refractive errors and concomitant strabismus: a systematic review and meta-analysis. Sci Rep. 2016;6:35177.

7. Zhu H, Yu JJ, Yu RB, Ding H, Bai J, Chen J, Liu H. Association between childhood strabismus and refractive error in Chinese preschool children. PLoS ONE. 2015;10:e0120720.

8. Wen G, Tarczy-Hornoch K, McKean-Cowdin R, Cotter SA, Borchert M, Lin J, et al. Prevalence of myopia, hyperopia, and astigmatism in non-Hispanic white and Asian children: multiethnic pediatric eye disease study. Ophthalmology. 2013;120: 2109-16.

9. BURIAN HM, SPIVEY BE. The surgical management of exodeviations. Trans Am Ophthalmol Soc. 1964;62:276-306.

10. Gezer A, Sezen F, Nasri N, Gözüm N. Factors influencing the outcome of strabismus surgery in patients with exotropia. J AAPOS. 2004;8:56-60.

11. Oh JY, Hwang JM. Survival analysis of 365 patients with exotropia after surgery. Eye (Lond). 2006;20:1268-72.

12. Chia A, Seenyen L, Long QB. Surgical experiences with twomuscle surgery for the treatment of intermittent exotropia. J AAPOS. 2006;10:206-11.

13. Stoller SH, Simon JW, Lininger LL. Bilateral lateral rectus recession for exotropia: a survival analysis. J Pediatr Ophthalmol Strabismus. 1994;31:89-92.

14. Kulp MT, Ying GS, Huang J, Maguire M, Quinn G, Ciner EB, et al. Associations between hyperopia and other vision and refractive error characteristics. Optom Vis Sci. 2014;91:383-9.
15. Colburn JD, Morrison DG, Estes RL, Li C, Lu P, Donahue SP. Longitudinal follow-up of hypermetropic children identified during preschool vision screening. J AAPOS. 2010;14: 211-5.

16. Yang M, Chen J, Shen T, Kang Y, Deng D, Lin X, et al. Clinical characteristics and surgical outcomes in patients with intermittent exotropia: a large sample study in South China. Medicine (Baltimore). 2016;95:e2590.

17. Lim SH, Hwang BS, Kim MM. Prognostic factors for recurrence after bilateral rectus recession procedure in patients with intermittent exotropia. Eye (Lond). 2012;26:846-52.

18. Kim MK, Kim US, Cho MJ, Baek SH. Hyperopic refractive errors as a prognostic factor in intermittent exotropia surgery. Eye (Lond). 2015;29:1555-60.

19. Zou D, Casafina C, Whiteman A, Jain S. Predictors of surgical success in patients with intermittent exotropia. J AAPOS. 2017;21:15-8.

20. Scattergood KD, Brown MH, Guyton DL. Artifacts introduced by spectacle lenses in the measurement of strabismic deviations. Am J Ophthalmol. 1983;96:439-48.

21. Von Noorden GK, Campos EC. Binocular vision and ocular motility: theory and management of strabismus. 6th ed. St. Louis: Mosby; 2002.

22. Iacobucci IL, Archer SM, Giles CL. Children with exotropia responsive to spectacle correction of hyperopia. Am J Ophthalmol. 1993;116:79-83.

23. Chung SA, Kim IS, Kim WK, Lee JB. Changes in exodeviation following hyperopic correction in patients with intermittent exotropia. J Pediatr Ophthalmol Strabismus. 2011;48: 278-84.

24. Adams WE, Leske DA, Hatt SR, Mohney BG, Birch EE, Weakley DR Jr, et al. Improvement in distance stereoacuity following surgery for intermittent exotropia. J AAPOS. 2008;12: $141-4$.

25. Holmes JM, Leske DA, Hatt SR, Brodsky MC, Mohney BG. Stability of near stereoacuity in childhood intermittent exotropia. J AAPOS. 2011;15:462-7.

26. Stathacopoulos RA, Rosenbaum AL, Zanoni D, Stager DR, McCall LC, Ziffer AJ, et al. Distance stereoacuity. Assessing control in intermittent exotropia. Ophthalmology. 1993;100:495-500.

27. O'Neal TD, Rosenbaum AL, Stathacopoulos RA. Distance stereo acuity improvement in intermittent exotropic patients following strabismus surgery. J Pediatr Ophthalmol Strabismus. 1995;32:3537. discussion 8

28. Yildirim C, Mutlu FM, Chen Y, Altinsoy HI. Assessment of central and peripheral fusion and near and distance stereoacuity in intermittent exotropic patients before and after strabismus surgery. Am J Ophthalmol. 1999;128:222-30.

29. Holmes JM, Birch EE, Leske DA, Fu VL, Mohney BG. New tests of distance stereoacuity and their role in evaluating intermittent exotropia. Ophthalmology. 2007;114:1215-20.

30. Simons K. Stereoacuity norms in young children. Arch Ophthalmol. 1981;99:439-45.

31. Fox R, Patterson R, Francis EL. Stereoacuity in young children. Invest Ophthalmol Vis Sci. 1986;27:598-600. 